\title{
Resident Immunity in Tissue Repair and Maintenance: The Zebrafish Model Coming of Age
}

\author{
Raquel Rua Martins 1,2, Pam S. Ellis 1,2, Ryan B. MacDonald ${ }^{2,3}$, Rebecca J. Richardson ${ }^{4}$ \\ and Catarina Martins Henriques ${ }^{1,2 *}$ \\ ' Department of Oncology and Metabolism, Medical School, University of Sheffield, Sheffield, United Kingdom, ${ }^{2}$ Bateson \\ Centre, University of Sheffield, Sheffield, United Kingdom, ${ }^{3}$ Department of Infection, Immunity and Cardiovascular Disease, \\ University of Sheffield, Sheffield, United Kingdom, ${ }^{4}$ School of Physiology, Pharmacology and Neuroscience, University \\ of Bristol, Bristol, United Kingdom
}

\section{OPEN ACCESS}

Edited by:

Eirini Trompouki,

Max-Planck-Institut für Immunbiologie und Epigenetik, Germany

Reviewed by:

Baubak Bajoghli,

Tübingen University Medical Center,

Germany

Jean-Pierre Levraud,

Institut Pasteur, France

${ }^{*}$ Correspondence:

Catarina Martins Henriques c.m.henriques@sheffield.ac.uk

Specialty section:

This article was submitted to

Cell Growth and Division,

a section of the journal

Frontiers in Cell and Developmental

Biology

Received: 29 September 2018

Accepted: 18 January 2019

Published: 05 February 2019

Citation:

Martins RR, Ellis PS,

MacDonald RB, Richardson RJ and Henriques CM (2019) Resident

Immunity in Tissue Repair and Maintenance: The Zebrafish

Model Coming of Age.

Front. Cell Dev. Biol. 7:12.

doi: 10.3389/fcell.2019.00012
The zebrafish has emerged as an exciting vertebrate model to study different aspects of immune system development, particularly due to its transparent embryonic development, the availability of multiple fluorescent reporter lines, efficient genetic tools and live imaging capabilities. However, the study of immunity in zebrafish has largely been limited to early larval stages due to an incomplete knowledge of the full repertoire of immune cells and their specific markers, in particular, a lack of cell surface antibodies to detect and isolate such cells in living tissues. Here we focus on tissue resident or associated immunity beyond development, in the adult zebrafish. It is our view that, with our increasing knowledge and the development of improved tools and protocols, the adult zebrafish will be increasingly appreciated for offering valuable insights into the role of immunity in tissue repair and maintenance, in both health and disease throughout the lifecourse.

Keywords: adult zebrafish (Danio Rerio), tissue resident immunity, tissue repair and regeneration, ageing, gut, heart, retina, brain

\section{INTRODUCTION}

It is becoming increasingly clear that the innate and adaptive immune systems play crucial roles in tissue maintenance and repair during health and disease. Studies in animal models are crucial to identifying complex functions of immunity in sometimes surprising aspects of biology. For example, it was discovered relatively recently that macrophages, previously thought of as purely cell debris-eating machines, promote fibrosis and scarring in mammals after an injury. Further, they have been identified as being crucial for tissue regeneration, directly communicating with epithelial cells in a variety of vertebrate models, reviewed elsewhere (Pott and Hornef, 2012; Ginhoux and Guilliams, 2016). Zebrafish are well placed as a model to decipher the complex functions of immune cells in tissue regeneration and other disease related processes due to their genetic tractability and the ease of live imaging. However, the majority of studies are largely limited to embryonic and larval stages due to their rapid, external development, genetic tractability, and transparent embryonic development. However, to best study tissue regeneration and human disease, fully differentiated tissues and organs are required. Here we put forward the adult zebrafish as a relevant and valid model for studying tissue immunity in health and disease throughout the whole animal's lifecourse. We highlight the recent advances in our knowledge of tissue immunity in adult zebrafish and the best tools currently available to study it. It is our view that our increasing knowledge and the ongoing development of tools and protocols are already making the adult zebrafish a valuable model 
offering insights into the role of immunity in tissue health throughout the lifecourse, and this model is likely to become more and more eminent in the future of the field, if we push forward for the continuous development of tools.

\section{Ontogeny of Tissue Immunity in Zebrafish}

It wouldn't make sense to delve into adult zebrafish tissue immunity before addressing their ontogeny. Unfortunately, though, this is where the problem starts. In the mouse, the most commonly used vertebrate immunology model, the origin of tissue resident or associated immune cells is generally well described, exemplified in (Bain et al., 2014; Ginhoux and Guilliams, 2016; Ferrero et al., 2018), whereas in the zebrafish, our knowledge is still largely incomplete.

In mice, extensive work over decades has shown that most tissues have resident immune cells, both innate (mainly macrophages and NK cells, depending on the tissue) and adaptive (T- and B-cells). The different flavors within these immune cells vary depending on tissue and disease status (Mowat et al., 2017; White et al., 2017). Amongst these, we know the most about macrophages. In mice, tissue-resident macrophages seem to derive from embryonic precursors that populate most tissues during embryogenesis, becoming a specialized, tissue-resident, self-renewing population in the adult (Hoeffel et al., 2012; Hashimoto et al., 2013; Yona et al., 2013; Hoeffel and Ginhoux, 2015). A well-known exception, at least in mice, is the gut. Recent work has shown that the macrophage population in the adult mouse gut is constantly re-populated by circulating monocytes, which then differentiate into mature macrophages and are maintained in situ (Bain et al., 2014; Bain and Mowat, 2014a,b). In zebrafish, our knowledge is more limited. Nevertheless, recent work by Alemany et al. has identified distinct signatures in resident immune cells in the adult zebrafish, using sophisticated single-cell sequencing and tracking analysis (Alemany et al., 2018). Their work shows that haematopoietic cells in the kidney marrow derive from a small set of multipotent embryonic progenitors. Surprisingly, the authors indicate that resident immune cells in the fin do not originate from haematopoietic stem cells and instead seem to arise either from epidermal and mesenchymal transdifferentiation, or from ectodermal ancestors similarly to mesenchymal cells. The origin and maintenance of resident immune cells remains to be fully elucidated in other organs such as the gut. Notwithstanding, the zebrafish model is also making key contributions to the understanding of tissue immunity in vertebrates, thanks to an impressive availability of transgenic reporter lines for different immune cells/inflammatory markers (see Table 1 for details). Seminal work has shown that, like in other vertebrates, zebrafish have a fully functional tissueassociated immunity, including T-cells, B-cells, macrophages, neutrophils, eosinophils, and mast cells (Moss et al., 2009; Renshaw and Trede, 2012; Nguyen-Chi et al., 2015; Pereiro et al., 2015; Dee et al., 2016), even if it is not yet determined whether they are resident in all tissues or not. Emerging data, however, is shedding light on the ontogeny of tissue immunity in zebrafish.
Recent work has shown that microglia, the specialized macrophages in the Central Nervous System (CNS); have different origins depending on the age of the animals. In the adult zebrafish, microglia derive from haematopoietic stem cells (HSCs) and not from primitive macrophages, which occurs only in early development (Ferrero et al., 2018). This has also been shown for adult zebrafish Langerhans cells in the skin and suggested to also be the case for liver, heart, gut and brain (He et al., 2018). Together, these elegant recent studies suggest that most zebrafish adult resident or associated immunity derives from the second wave of hematopoiesis, mainly from the ventral wall of the dorsal aorta (VDA region), and not from erythromyeloid progenitors (EMPs) as previously thought. This is also emerging as the current model in mammalian systems (Sheng et al., 2015) although there are still uncertainties and some controversy in the field (Perdiguero et al., 2015).

Crucially, recent work is showing that, more than ontogeny, tissue immunity seems to be particularly dictated by the tissue in which it resides. There are key tissues in adult zebrafish that are being intensely investigated and multiple studies highlight that the role of immunity in tissue repair and maintenance is largely conserved in zebrafish. Key examples where this has been shown are in the heart, gut, brain, and retina.

\section{Selected Examples of Tissue Immunity in Adult Zebrafish \\ Heart}

Recent years have seen many studies identify crucial and perhaps surprising roles for immune cell populations in the heart in homeostasis and disease, although much remains to be discovered. A recent study in mice indicated a remarkable role for resident cardiac macrophages in the distal atrioventricular node where they make direct connections to cardiomyocytes via Connexin 43 and facilitate electrical conductance (Hulsmans et al., 2017). In zebrafish, we currently know very little about cardiac macrophages under homeostatic conditions although our own experience has revealed a population of immune cells, labeled with L-plastin and transgenic markers of macrophages (see Table 1), is present in the unwounded heart and recent work suggests these may be derived from HSCs (see above; Figure 1). Recently, many studies have shown important contributions of different immune cell lineages in response to cardiac injury and disease in mammalian models. In particular, vital roles have been suggested for macrophages in complete regeneration of the neonatal mouse heart (Aurora and Olson, 2014; Lavine et al., 2014). However, the inflammatory response in the adult zebrafish heart has been less well characterized. Recent studies revealed that immune cells are recruited to the heart following cryoinjury of the ventricle in adult zebrafish (Schnabel et al., 2011). Two recent reports have also shown that macrophages are required for cardiomyocyte proliferation and therefore regeneration in the heart of adult zebrafish (de Preux Charles et al., 2016; Lai et al., 2017). Our own experience suggests that all immune cell lineages that we were able to analyse are recruited to the heart after injury and whereas roles can be assigned for macrophages of the innate immune system, the precise roles for other cell types remain more 


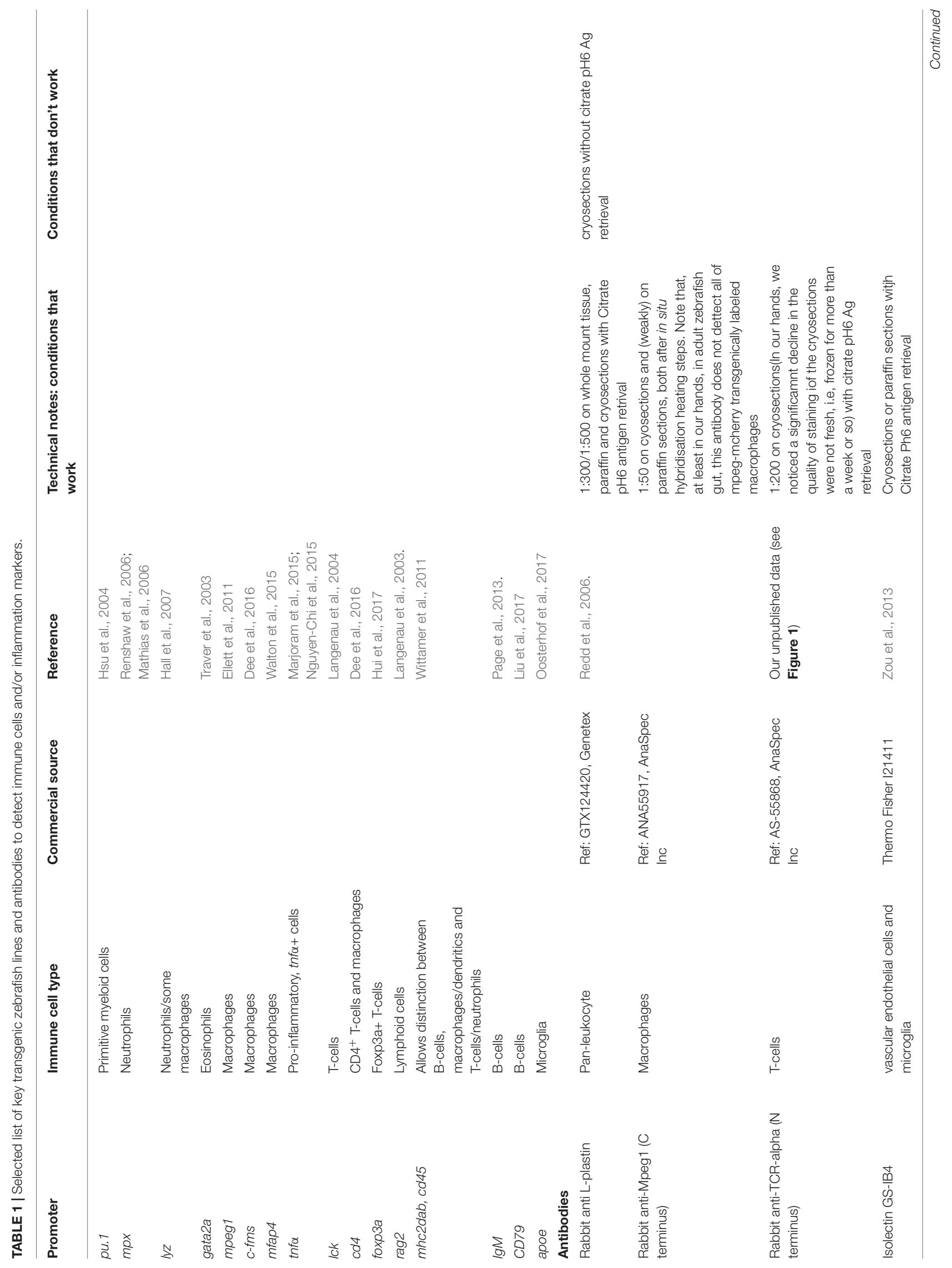




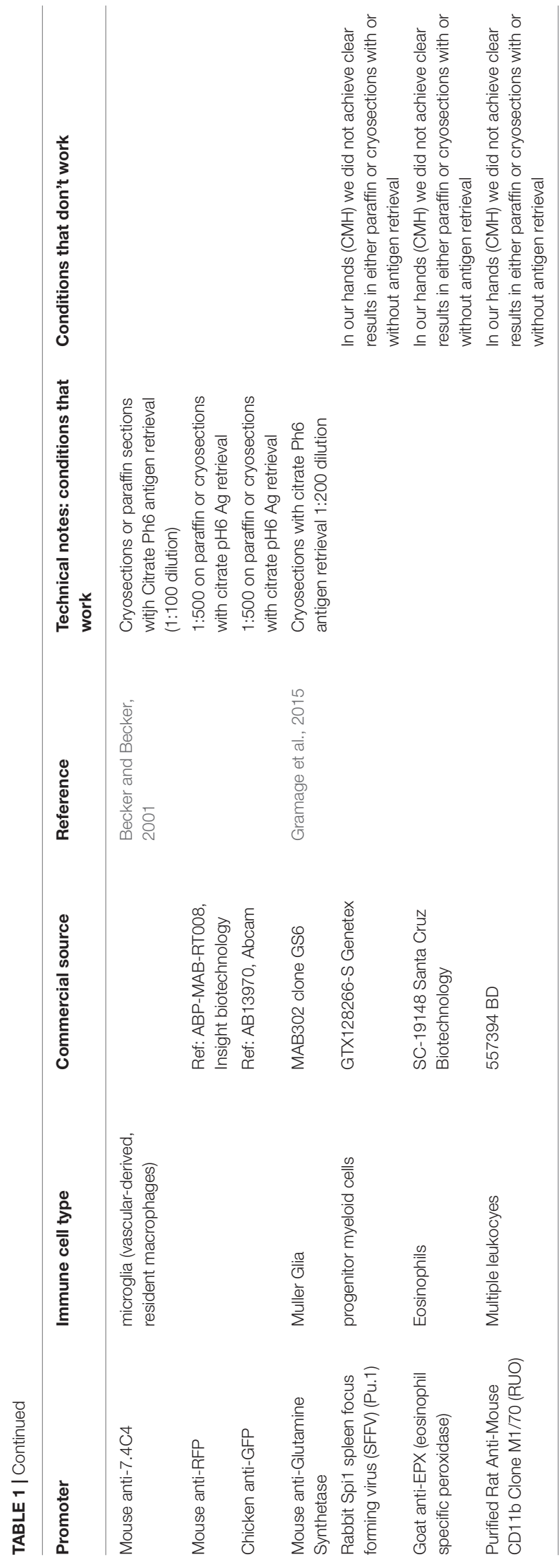

of a mystery (RJR, unpublished). However, another recent report has shown important roles for a Treg subset of zebrafish T-cells in promoting regeneration in a number of different tissues including the heart (Hui et al., 2017), suggesting intriguing and important roles for different immune cell populations in varying aspects of regeneration and disease remain to be elucidated.

\section{Gut}

The gut can be considered the biggest compartment of the immune system, and it is constantly exposed to multiple foreign antigens, which it must distinguish from harmless dietary proteins and the resident microbiota (Mowat, 2018). When this goes wrong, the immune system can "misfire" and contribute to chronic inflammatory disorders such as Inflammatory Bowel Disease (IBD) (Bain and Mowat, 2014b; Mowat et al., 2017; Andrews et al., 2018; Corridoni et al., 2018; Liu et al., 2018) and age-associated gut degeneration (Man et al., 2014; Sato et al., 2015; Soenen et al., 2016). Additionally, gut immunity is essential for the steady-state epithelial renewal (Andrews et al., 2018) that, similarly to humans, occurs roughly every 3 days in adult zebrafish (Wallace et al., 2005; Crosnier et al., 2006). Recovery after DSS intestinal injury has also been shown to be dependent on Myd88 signaling in myeloid cells (Malvin et al., 2012). Adult zebrafish are also showing great promise as a model to study gut inflammation and repair in health and disease (Marjoram and Bagnat, 2015 Brugman, 2016), including ageing (Henriques et al., 2013; Carneiro et al., 2016, Martins et al., 2018). These works have shown that, similarly to humans, critical aspects of gut homeostasis become compromised with ageing in zebrafish, namely increased permeability, inflammation and telomere-dependent cellular senescence.

Thanks to the development of key transgenic reporter lines and antibodies (Table 1), adult zebrafish gut has been shown to be populated by abundant T-cells, B cells, mast cells, macrophages, and dendritic cells in the normal steady-state context (CMH unpublished data and others) (Wittamer et al., 2011; Lewis et al., 2014), similarly to mammalian vertebrates (Figure 1). Importantly, macrophage M1 and M2-like subsets have also been identified in zebrafish, thanks to the development of mpeg-mcherry-TNF $\alpha$-GFP double transgenic line. Using this line, M1 macrophages were characterized by high TNF $\alpha$-GFP expression (mpeg+TNF $\alpha+$ ), as well as expression of TNF $\beta$, IL-1 $\beta$, and IL-6 (Nguyen-Chi et al., 2015), which are wellknown markers of M1-like macrophages in mammals. Moreover, these subsets were shown to respond to injury similarly to human macrophage subsets. Additionally, Il-1 $\beta$ reporter lines have recently been developed allowing visualization of cells expressing this pro-inflammatory cytokine (Hasegawa et al., 2017; Ogryzko et al., 2018). What is very different in the zebrafish gut, however, is the absence, as far as reported, of defined intestinal crypts and Peyer's patches (Ng et al., 2005; Cheng et al., 2016). Despite the absence of Peyer's patches there is a clear distribution of leukocytes along the adult zebrafish gut, lining the enterocytes, which could be considered analogous to the mucosal associated lymphoid tissue (MALT). The apparent absence of secondary lymphoid structures though, means that we still do not understand fully how antigen presentation occurs in zebrafish 


\section{Adult zebrafish imaging of tissue immunity}

$$
\text { Beart Skin }
$$
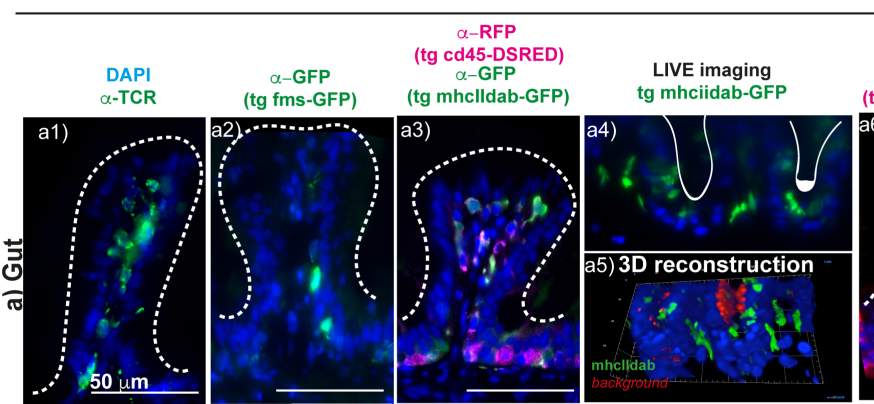

$$
\alpha-R F P
$$

(tg mpeg-mcherry) $\quad \alpha$-L-plastin
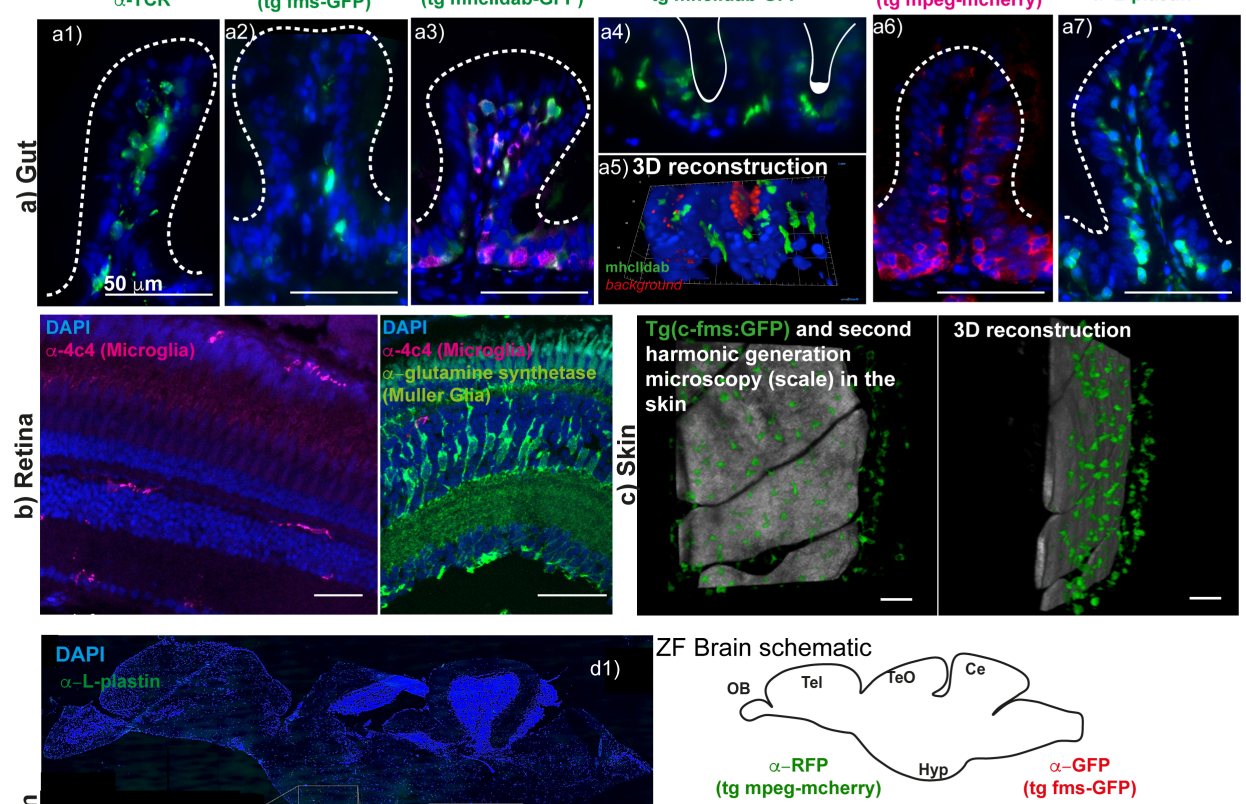

ZF Brain schematic
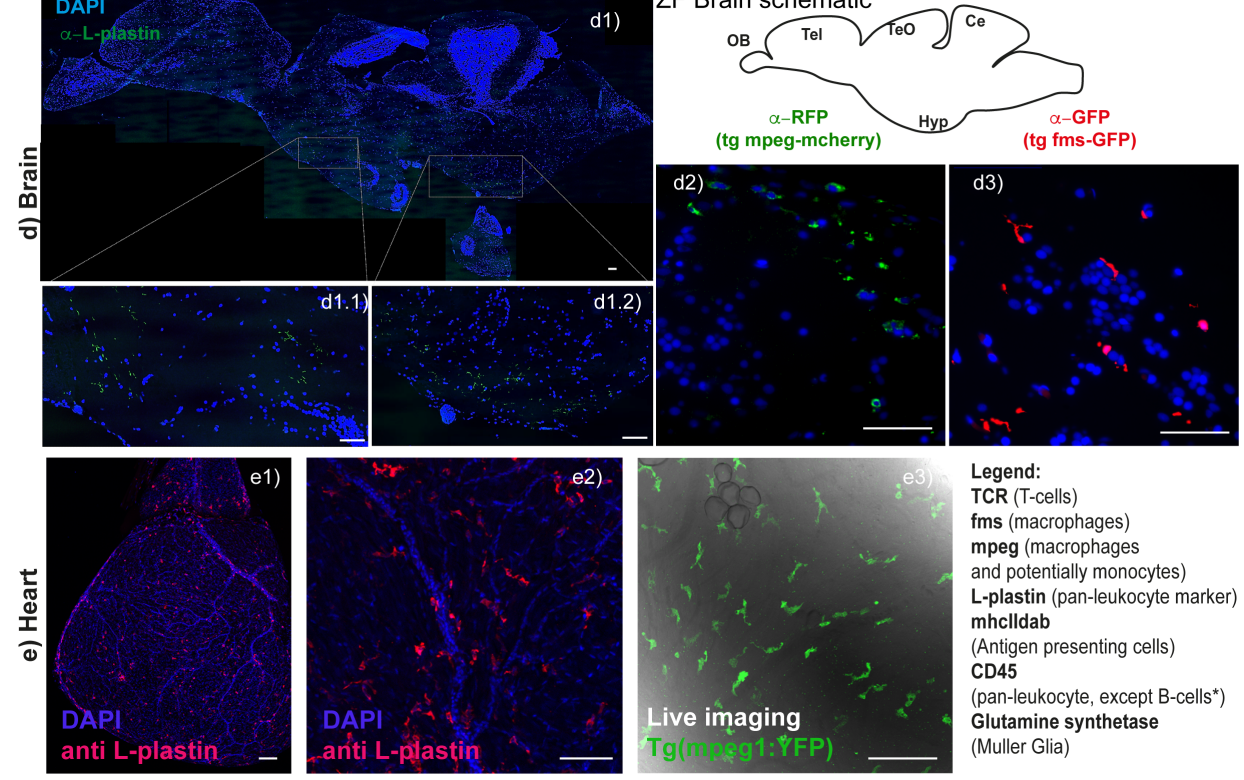

FIGURE 1 | Imaging tissue immunity in adult Zebrafish. (a) CMH unpublished images showing examples of cryo/paraffin sections of adult Zebrafish gut, followed by immune fluorescence of selected antigens (see table 1 for antibody details), all counterstained with DAPI for cell nuclei detection and imaged in a Deltavision light microscope. (a1) T-cells are stained with anti-TCR alpha antibody (a2) macrophages in Tg(fms-GFP) animals (Dee et al., 2016) are detected with an anti-GFP antibody; (a3) multiple leukocyte lineages can be detected using anti-RFP and anti-GFP antibodies in a double transgenic line for CD45-DSRED and mhclldab-GFP respectively (Wittamer et al., 2011). Single red cells are neutrophils/T-cells, double green and red cells are macrophages/dendritic cells, single green cells are B-cells, since, in this line, the CD45 promoter used is not active in B-cells; (a4) and (a5) Adult Zebrafish gut can be imaged in Light sheet microscopy for short durations, following dissection and gentle embedding in low percentage agarose in E3 media; (a6) macrophages are detected with an anti-RFP antibody in Tg(mpeg1: mCherryCAAX) (Ellett et al., 2011; Ogryzko et al., 2018) animals; (a7) leukocytes are stained with L-plastin (aka LCP-1) and these can $n=$ be seen lining the gut epithelia cells (enterocytes). (b) RBM unpublished images showing selected cryosections of adult Zebrafish retina, imaged by confocal microscopy followed by immune fluorescence of selected antigens (see table 1 for antibody details), all counterstained with DAPI for cell nuclei detection. (b1) Microglia are detected with an anti-4c4 antibody and can be seen dispersed throughout the tissue, displaying a simple ramified state. Upon insult these microglia rapidly migrate and engulf damaged cells or debris. (b2) Muller Glia can also be detected in the retina using an anti-glutamine synthetase antibody. (c) RJR unpublished images of adult zebrafish skin where macrophages can be live-imaged, shown here using the $\mathrm{Tg}(c-f m s-G F P)$ line and using second harmonic generation microscopy to identify 


\section{FIGURE 1 | Continued}

the scale surface. (d) RRM unpublished images showing paraffin sections of adult Zebrafish brain, imaged using a Deltavision light microscope or by confocal microscopy; (d1) multiple-panel reconstitution of a whole adult brain, imaged by confocal microscopy, stained with anti-L-plastin, which marks all microglia in the brain. Microglia in the adult brain can also be detected for example using the (d1) Tg(mpeg1:mCherryCAAX) line (Ellett et al., 2011; Ogryzko et al., 2018) or (d2) the Tg(fms-GFP) line (Dee et al., 2016). Sections (d1) and (d2) show the diencephalon. (e) RJR unpublished images showing whole mount immunostaining of an entire heart (e1) or the surface of the ventricle (e2) with an anti-L plastin antibody and imaged with confocal microscopy. Live imaging using confocal microscopy of an unwounded adult zebrafish heart reveals cardiac macrophages using a $\operatorname{Tg}(m p e g 1: Y F P)$ line (e3).

(Lewis et al., 2014). MHC class II-expressing, antigen presenting cells (APCs), namely macrophages, dendritics (DCs) and B-cells are, however, described and appear to function similarly to their mammalian counterparts (Lugo-Villarino et al., 2010; Wittamer et al., 2011, Lewis et al., 2014; Dee et al., 2016). See Table 1 for further details and references.

Nevertheless, and importantly, the protective immune status of the gut has also been shown to occur in zebrafish, and this has been largely attributed to the secretion of IL-10 by CD41+foxp3+ Treg-like T-cells in the gut, once again showing that these sophisticated immune-regulatory elements are already evolved in teleost fish (Dee et al., 2016; Hui et al., 2017). It has also been shown that in adult zebrafish, the gut is rapidly populated by eosinophils upon parasitic infections (Balla et al., 2010), highlighting another conserved response to infection in the gut.

\section{Brain}

The neuro-immunology field has been growing in recent years, highlighting the complex crosstalk between the immune system and the central nervous system (CNS) and how this plays a key role in maintaining brain homeostasis, reviewed in (Oosterhof et al., 2015). Although mammalian models are more prominent at the moment, the zebrafish model is starting to gain traction, particularly due to its genetic and imaging amenity, small size and relatively low maintenance costs (de Abreu et al., 2018).

As in mammals (Cuadros and Navascues, 1998; Ginhoux et al., 2010), microglia are the tissue-resident immune cells in the zebrafish brain (Xu et al., 2015). In zebrafish, primitive microglia originate from yolk sac-derived macrophages that migrate to the cephalic mesenchyme and then invade the brain, being detectable from $60 \mathrm{~h}$ post-fertilization (hpf) (Herbomel et al., 2001). Only more recently it was recognized that these are not the definitive microglia observed in adulthood. As described above, an elegant recent study (Ferrero et al., 2018), showed the existence of a second wave of re-population of the brain's microglia, which originate from HSCs - a process that occurs between 2 weeks and 3 months of age. Microglia can be detected by L-plastin (or LCP-1), a pan-leukocyte marker (Redd et al., 2006; Cvejic et al., 2008; Mathias et al., 2010; Van Houcke et al., 2017), by macrophage-expressed gene 1 (mpeg1), labeling all mononuclear phagocytes (Ellett et al., 2011; Wittamer et al., 2011), by the 4c4 antibody (Becker and Becker, 2001)as well as Apolipoprotein E (ApoE) [Oosterhof et al. (2015), see Table 1; Figure 1]. Note that, although ApoE specifically labels microglia if compared with the other markers mentioned, it has also been shown to be expressed astrocytes (Boyles et al., 1985; Poirier et al., 1991; Xu et al., 2006), oligodendrocytes (Stoll et al., 1989), as well as by some neurons albeit at lower levels (Han et al., 1994; Achariyar et al., 2016). Additionally, cells in the choroid plexus as well as smooth muscle cells in blood vessels also seem to express ApoE (Xu et al., 2006). Thus, in order to ensure specific labeling of microglia, ApoE should be used in combination with another leukocyte or macrophage marker.

Microglia have been described as ramified cells that constantly sense the environment searching for physiological disturbances in the surroundings (Oosterhof et al., 2015). Like in mammals (Lucin and Wyss-Coray, 2009), adult zebrafish microglia proliferate and migrate to the injury or inflammation site ('gliosis'), upon activation in response to a stab lesion (Kroehne et al., 2011; Kyritsis et al., 2012), excitotoxin injection (Skaggs et al., 2014) or nitroreductase (NTR)-mediated neuronal ablation (Oosterhof et al., 2017). Also, there is an increased number of L-plastin ${ }^{+}$cells in response to optic nerve injury in both young (5 months) and older (22-24 months) zebrafish, but this is decelerated in the old fish, suggesting age-related dysfunctional immune response in ageing (Van Houcke et al., 2017). Once activated, microglia change their appearance from a ramified to an amoeboid shape (Svahn et al., 2013). These immune cells have the important function of clearing cellular debris, such as dead or damaged neurons, by phagocytosis (Peri and Nusslein-Volhard, 2008); and, when activated can release antiand pro- inflammatory cytokines, at least in mice primary microglia cultures (Cai et al., 2017). To our knowledge, microglia inflammatory cytokine release remains to described for zebrafish, despite extensive characterization of other aspects of zebrafish microglia (van Ham et al., 2014). Peripheral immune cells can infiltrate the CNS in cases of Blood Brain Barrier (BBB) alterations, such as those observed in Multiple Sclerosis (MS) or cerebral ischemia (Holtmaat and Caroni, 2016), in particular, infiltration of monocytes or perivascular macrophages has been described in mammals (Lucin and Wyss-Coray, 2009). Similarly, upon NTR-induced cell death, peripheral macrophagelike cells infiltrate the embryonic zebrafish brain, contributing to the first inflammatory response (van Ham et al., 2014). In opposition, it has been reported that no major infiltration of periphery macrophages occurs in the brain after neuronal ablation (Oosterhof et al., 2017). Thus, more studies are needed to address this question. More surprisingly, T cells were reported to infiltrate the brain in a mouse model of ALS (SOD1 ${ }^{\text {G93A }}$ ) during progression of the disease (Chiu et al., 2008) and to invade the human brain in Parkinson's Disease (PD) (Brochard et al., 2009). Also, CD ${ }^{+} \mathrm{T}$ cells and $\mathrm{B}$ cells have been detected in the brain of patients with MS, and this is thought to contribute to inflammation in the CNS (Jelcic et al., 2018). To our knowledge, so far, there are no studies reporting the presence of $\mathrm{T}$ cells or $\mathrm{B}$ 
cells in zebrafish brain. Zebrafish Treg-like (zTreg) cells seem to move towards damaged sites in CNS, such as retina and spinal cord, contributing to regeneration; however, the brain was not explored in this study (Hui et al., 2017). Moreover, it remains unknown whether neutrophils invade the adult zebrafish brain in contexts of severe inflammation. Neutrophils were found in the brain of a nlrc3-like mutant model zebrafish embryo, where there is a systemic inflammation (Shiau et al., 2013). However, no recruitment of neutrophils was observed after injury either in the embryo brain (van Ham et al., 2014) or peripheral nervous system (Pope and Voigt, 2014). Additionally, Goldshmit et al. reported to rarely find neutrophils at the injury site after spinal cord transection in adult zebrafish (Goldshmit et al., 2012). Unfortunately, though, other studies have yet to be reported for adult zebrafish to help clarify this matter.

\section{Retina}

The retina is viewed as a unique "window" into the brain and is one of the most established systems to study neural development and disease processes in the CNS (London et al., 2013). The zebrafish retina is a true vertebrate retina as it has the same organisation and contains largely the same types of neurons and glial cells as the human eye. The innate immune system in the zebrafish retina is composed of two major types of glial cell, the Müller glia (MG) and the microglia (Figure 1). The mammalian retina also houses astrocytes that will contribute to immunity. However, their presence in the zebrafish CNS, including the retina, remains unclear (Lyons and Talbot, 2014). The MG and microglia will contribute to the maintenance of homeostasis, phagocytose debris and are critical for tissue repair (Reichenbach and Bringmann, 2013). MG are the most abundant glial cell in the tissue, have a fixed radial morphology which allows them to contact surrounding neurons (Jadhav et al., 2009) and can modulate innate retinal immunity (Kumar et al., 2013; Vecino et al., 2016). Retina microglia are migratory, as in the brain, and survey the tissue for damage and debris (Silverman and Wong, 2018). Crosstalk between these two glial cell types may mediate their response to damage and injury by coordinating inflammation (Wang et al., 2011, 2014). Activated MG and microglia are associated with almost every pathological condition in the retina (Bringmann et al., 2006; Silverman and Wong, 2018). This includes retinal degenerative conditions, such as age related macular degeneration and diabetic retinopathies (Ramirez et al., 2017). The zebrafish is an established model for studying cellular and molecular mechanisms underlying many ocular diseases (Gestri et al., 2012). However, linking immunity with confounding factors for disease, such as ageing, remain challenging in many models. A recent study in zebrafish has shown that there is progressive degeneration of photoreceptors with age when interfering with Crumbs, a gene family linked with human retinal degeneration (Fu et al., 2018). However, the contribution of the innate immune system to degeneration and pathologies of disease remains largely unknown.

After damage the innate immune systems plays a key role in the phagocytosis of debris and removal of dead or dying cells (Kumar et al., 2013). However, in the zebrafish retina after damage or disease the MG will generate neurons to restore vision
(Hitchcock and Raymond, 2004). This is an area of intense study and the molecular mechanisms regulating it are beginning to be identified (Goldman, 2014), yet the role of microglia in these processes is not clear (Mitchell et al., 2018). By imaging the glial dynamics in real time in vivo in the zebrafish retina, microglia have been shown to change their morphology to the activated state and maintain this activation after regeneration is complete, potentially to ensure correct retinal function is re-established (Mitchell et al., 2018). Further, by pairing the imaging capacity of the zebrafish with the ease to which they can be treated with pharmacological inhibitors a recent study investigated roles of the innate immune system during rod photoreceptor regeneration (White et al., 2017). They show that the role of microglia is to regulate MG responsiveness to cell death, and thereby control neuronal regeneration kinetics. Further, immunosuppression can either inhibit or accelerate photoreceptor regeneration kinetics depending on the timing of treatment (White et al., 2017). Thus, utilizing the precise advantages of the zebrafish, paired with the well-characterized retina, makes this an exciting model to study the resident immune system in retinal disease and regeneration.

\section{CONCLUDING REMARKS}

Despite multiple advances in developing reporter transgenic lines marking different types of immune cell lineages in zebrafish, there are still multiple sub-types of immune cells we have no markers for or antibodies available e.g., mast cells. Nevertheless, advances in single-cell sequencing technology have already enabled the identification of specific immune subsets, such as different subtypes of NK cells (Carmona et al., 2017; Tang et al., 2017) and innate lymphoid cells (ILCs) (Hernandez et al., 2018), which have contributed to the understanding of the similarities and differences between zebrafish and human immune subsets. Despite the overall similarity between human and zebrafish immune subsets, highlighted here, there are key differences, which are important to keep in mind, reviewed elsewhere (Trede et al., 2004; Renshaw and Trede, 2012, Kanwal et al., 2014). The first obvious difference is that during the first week of zebrafish development, this organism relies entirely on an innate immune system (Lam et al., 2004), a difference which has been extensively used to understand the relative contribution of innate versus adaptive immunity in response to different bacterial, viral, and fungal pathogens (Meijer and Spaink, 2011; Meijer et al., 2014). Another key difference is the absence, at least not reported thus far, of secondary lymphoid organs in zebrafish. Moreover, the zebrafish does not have a bone marrow, and instead, T-, B- as well as myeloid cells are present in the spleen and head kidney, which act as the zebrafish equivalent of bone marrow. There are also key differences in zebrafish immune receptors and/or response to specific ligands reviewed in (Kanwal et al., 2014) and this is contributed to by the gene duplication detected in many of the zebrafish genes (Lu et al., 2012) An example are the novel immune-type receptors (NITRs), which appear to be homologues of mammalian NK-like receptors and seem to also have homologous functions (Yoder et al., 2010). Additionally, despite the fact that most of Toll Like receptors 
have been described in zebrafish, there are key differences such as the fact that Tlr4 is not involved in sensing LPS. Indeed, in zebrafish, LPS signals via a Tlr4- and MyD88-independent manner (Sepulcre et al., 2009). Nevertheless, zebrafish still respond to lipopolysaccharide (LPS), and careful analysis has shown that the overall response to LPS stimulation at the level of gene transcription is highly conserved with that of mammals (Forn-Cuni et al., 2017).

We have highlighted in Table 1 the working tools available as well as some antibodies that we have tested but have failed to get to work. We believe this will be a valuable starting point for future researchers wanting to use zebrafish to study tissue immunity.

In summary, we can clearly identify microglia, macrophages (including distinguishing a pro-inflammatory phenotype), T-cells, B-cells, and neutrophils in tissues using a combination of transgenic lines and antibodies. It will be particularly important to develop these techniques further if we are to improve our live imaging capability, but also the ability to detect multiple immune lineages in the same tissue without requiring crossing multiple transgenic lines, which dramatically increases the time and cost of experiments. Unfortunately, we are still missing transgenic reporters and/or antibodies for some sub-types of T-cells (e.g., Th1, Th2, cytotoxic, and NKT), NK-cells and mast cells.

We hope that the studies highlighted here show how zebrafish can offer an incredible tool to study immunity and its role in tissue repair and maintenance, across the lifecourse, in a time and cost-efficient manner, and how it can improve so much more with the continuous investment, not only of this scientific community, which is growing, but also of commercial companies, particularly in the development and validation of zebrafishspecific antibodies.

\section{REFERENCES}

Achariyar, T. M., Li, B., Peng, W., Verghese, P. B., Shi, Y., McConnell, E., et al. (2016). Glymphatic distribution of CSF-derived apoE into brain is isoform specific and suppressed during sleep deprivation. Mol. Neurodegener. 11:74. doi: 10.1186/s13024-016-0138-8

Alemany, A., Florescu, M., Baron, C. S., Peterson-Maduro, J., and van Oudenaarden, A. (2018). Whole-organism clone tracing using single-cell sequencing. Nature 556, 108-112. doi: 10.1038/nature25969

Andrews, C., McLean, M. H., and Durum, S. K. (2018). Cytokine tuning of intestinal epithelial function. Front. Immunol. 9:1270. doi: 10.3389/fimmu.2018. 01270

Aurora, A. B., and Olson, E. N. (2014). Immune modulation of stem cells and regeneration. Cell Stem Cell 15, 14-25. doi: 10.1016/j.stem.2014.06.009

Bain, C. C., Bravo-Blas, A., Scott, C. L., Perdiguero, E. G., Geissmann, F., Henri, S., et al. (2014). Constant replenishment from circulating monocytes maintains the macrophage pool in the intestine of adult mice. Nat. Immunol. 15, 929-937. doi: $10.1038 /$ ni.2967

Bain, C. C., and Mowat, A. M. (2014a). Macrophages in intestinal homeostasis and inflammation. Immunol. Rev. 260, 102-117. doi: 10.1111/imr.12192

Bain, C. C., and Mowat, A. M. (2014b). The monocyte-macrophage axis in the intestine. Cell. Immunol. 291, 41-48. doi: 10.1016/j.cellimm.2014.03.012

Balla, K. M., Lugo-Villarino, G., Spitsbergen, J. M., Stachura, D. L., Hu, Y., Bañuelos, K., et al. (2010). Eosinophils in the zebrafish: prospective isolation, characterization, and eosinophilia induction by helminth determinants. Blood 116, 3944-3954. doi: 10.1182/blood-2010-03-267419

\section{ETHICS STATEMENT}

This study complied with the Animals (Scientific Procedures) Act 1986 using Home Office approved licenses [PPL numbers: 30/3318 (RJR), 70/8681 (CMH), and 40/3727 (RBM)]. The licenses and protocols were ethically reviewed and approved by each local Animal Welfare and Ethical Review Body (AWERB) (University of Sheffield and University of Bristol). Both Universities are signatories of the Understanding Animal Research Concordat on Openness and as Signatories to the Concordat have agreed to be more open about their use of animals in research, and to abide by the four commitments.

\section{AUTHOR CONTRIBUTIONS}

$\mathrm{CMH}, \mathrm{RRM}, \mathrm{RBM}$, and RJR contributed equally to the writing of the manuscript, figure, and table. PSE contributed to the development of key techniques illustrated in Figure $\mathbf{1}$ and contributed to Table 1 .

\section{FUNDING}

$\mathrm{CMH}$ was funded by a Sheffield University Vice Chancellor's Research Fellowship and the Sir. Henry Dale Fellowship by the Welcome Trust and Royal Society. RRM was funded by a University of Sheffield, United Kingdom, Ph.D. studentship. PSE was funded by a PDRA salary included in CMH Sir Henry Dale Fellowship (The Welcome Trust and Royal Society). RBM was funded by a JG Graves Medical Research Fellowship and a Welcome Trust Seed Award. RJR was funded by the British Heart Foundation (Intermediate Fellowship).

Becker, T., and Becker, C. G. (2001). Regenerating descending axons preferentially reroute to the gray matter in the presence of a general macrophage/microglial reaction caudal to a spinal transection in adult zebrafish. J. Comp. Neurol. 433, 131-147. doi: 10.1002/cne.1131

Boyles, J. K., Pitas, R. E., Wilson, E., Mahley, R. W., and Taylor, J. M. (1985). Apolipoprotein $\mathrm{E}$ associated with astrocytic glia of the central nervous system and with nonmyelinating glia of the peripheral nervous system. J. Clin. Invest. 76, 1501-1513. doi: 10.1172/JCI112130

Bringmann, A., Pannicke, T., Grosche, J., Francke, M., Wiedemann, P., Skatchkov, S. N., et al. (2006). Muller cells in the healthy and diseased retina. Prog. Retin. Eye Res. 25, 397-424. doi: 10.1016/j.preteyeres.2006. 05.003

Brochard, V., Combadiere, B., Prigent, A., Laouar, Y., Perrin, A., Beray-Berthat, V., et al. (2009). Infiltration of $\mathrm{CD}^{+}$lymphocytes into the brain contributes to neurodegeneration in a mouse model of Parkinson disease. J. Clin. Invest. 119, 182-192. doi: 10.1172/JCI36470

Brugman, S. (2016). The zebrafish as a model to study intestinal inflammation. Dev. Comp. Immunol. 64, 82-92. doi: 10.1016/j.dci.2016.02.020

Cai, Q., Li, Y., and Pei, G. (2017). Polysaccharides from Ganoderma lucidum attenuate microglia-mediated neuroinflammation and modulate microglial phagocytosis and behavioural response. J. Neuroinflammation 14:63. doi: 10. 1186/s12974-017-0839-0

Carmona, S. J., Teichmann, S. A., Ferreira, L., Macaulay, I. C., Stubbington, M. J., Cvejic, A., et al. (2017). Single-cell transcriptome analysis of fish immune cells provides insight into the evolution of vertebrate immune cell types. Genome Res. 27, 451-461. doi: 10.1101/gr.207704.116 
Carneiro, M. C., Henriques, C. M., Nabais, J., Ferreira, T., Carvalho, T., and Ferreira, M. G. (2016). Short telomeres in key tissues initiate local and systemic aging in zebrafish. PLoS Genet. 12:e1005798. doi: 10.1371/journal.pgen.1005798

Cheng, D., Shami, G. J., Morsch, M., Chung, R. S., and Braet, F. (2016). Ultrastructural mapping of the zebrafish gastrointestinal system as a basis for experimental drug studies. Biomed Res. Int. 2016:8758460. doi: 10.1155/2016/ 8758460

Chiu, I. M., Chen, A., Zheng, Y., Kosaras, B., Tsiftsoglou, S. A., Vartanian, T. K., et al. (2008). T lymphocytes potentiate endogenous neuroprotective inflammation in a mouse model of ALS. Proc. Natl. Acad. Sci. U.S.A. 105, 17913-17918. doi: 10.1073/pnas.0804610105

Corridoni, D., Chapman, T., Ambrose, T., and Simmons, A. (2018). Emerging mechanisms of innate immunity and their translational potential in inflammatory bowel disease. Front. Med. 5:32. doi: 10.3389/fmed.2018.00032

Crosnier, C., Stamataki, D., and Lewis, J. (2006). Organizing cell renewal in the intestine: stem cells, signals and combinatorial control. Nat. Rev. Genet. 7, 349-359. doi: 10.1038/nrg1840

Cuadros, M. A., and Navascues, J. (1998). The origin and differentiation of microglial cells during development. Prog. Neurobiol. 56, 173-189. doi: 10.1016/ S0301-0082(98)00035-5

Cvejic, A., Hall, C., Bak-Maier, M., Flores, M. V., Crosier, P., Redd, M. J., et al. (2008). Analysis of WASp function during the wound inflammatory responselive-imaging studies in zebrafish larvae. J. Cell Sci. 121(Pt 19), 3196-3206. doi: $10.1242 /$ jcs. 032235

de Abreu, M. S., Giacomini, A. C. V. V., Zanandrea, R., Dos Santos, B. E., Genario, R., de Oliveira, G. G., et al. (2018). Psychoneuroimmunology and immunopsychiatry of zebrafish. Psychoneuroendocrinology 92, 1-12. doi: 10 . 1016/j.psyneuen.2018.03.014

de Preux Charles, A. S., Bise, T., Baier, F., Marro, J., and Jazwinska, A. (2016). Distinct effects of inflammation on preconditioning and regeneration of the adult zebrafish heart. Open Biol. 6:160102. doi: 10.1098/rsob.160102

Dee, C. T., Nagaraju, R. T., Athanasiadis, E. I., Gray, C., Fernandez Del Ama, L., Johnston, S. A., et al. (2016). CD4-transgenic zebrafish reveal tissue-resident $\mathrm{Th} 2$ - and regulatory $\mathrm{T}$ cell-like populations and diverse mononuclear phagocytes. J. Immunol. 197, 3520-3530. doi: 10.4049/jimmunol. 1600959

Ellett, F., Pase, L., Hayman, J. W., Andrianopoulos, A., and Lieschke, G. J. (2011). mpeg1 promoter transgenes direct macrophage-lineage expression in zebrafish. Blood 117, e49-e56. doi: 10.1182/blood-2010-10-314120

Ferrero, G., Mahony, C. B., Dupuis, E., Yvernogeau, L., Di Ruggiero, E., Miserocchi, M., et al. (2018). Embryonic microglia derive from primitive macrophages and are replaced by cmyb-dependent definitive microglia in zebrafish. Cell Rep. 24, 130-141. doi: 10.1016/j.celrep.2018.05.066

Forn-Cuni, G., Varela, M., Pereiro, P., Novoa, B., and Figueras, A. (2017). Conserved gene regulation during acute inflammation between zebrafish and mammals. Sci. Rep. 7:41905. doi: 10.1038/srep41905

Fu, J., Nagashima, M., Guo, C., Raymond, P. A., and Wei, X. (2018). Novel animal model of crumbs-dependent progressive retinal degeneration that targets specific cone subtypes. Invest. Ophthalmol. Vis. Sci. 59, 505-518. doi: 10.1167/iovs.17-22572

Gestri, G., Link, B. A., and Neuhauss, S. C. (2012). The visual system of zebrafish and its use to model human ocular diseases. Dev. Neurobiol. 72, 302-327. doi: 10.1002/dneu.20919

Ginhoux, F., Greter, M., Leboeuf, M., Nandi, S., See, P., Gokhan, S., et al. (2010). Fate mapping analysis reveals that adult microglia derive from primitive macrophages. Science 330, 841-845. doi: 10.1126/science.1194637

Ginhoux, F., and Guilliams, M. (2016). Tissue-resident macrophage ontogeny and homeostasis. Immunity 44, 439-449. doi: 10.1016/j.immuni.2016.02.024

Goldman, D. (2014). Muller glial cell reprogramming and retina regeneration. Nat. Rev. Neurosci. 15, 431-442. doi: 10.1038/nrn3723

Goldshmit, Y., Sztal, T. E., Jusuf, P. R., Hall, T. E., Nguyen-Chi, M., and Currie, P. D. (2012). Fgf-dependent glial cell bridges facilitate spinal cord regeneration in zebrafish. J. Neurosci. 32, 7477-7492. doi: 10.1523/JNEUROSCI.0758-12. 2012

Gramage, E., D’Cruz, T., Taylor, S., Thummel, R., and Hitchcock, P. F. (2015). Midkine-a protein localization in the developing and adult retina of the zebrafish and its function during photoreceptor regeneration. PLoS One 10:e0121789. doi: 10.1371/journal.pone.0121789
Hall, C., Flores, M. V., Storm, T., Crosier, K., and Crosier, P. (2007). The zebrafish lysozyme $\mathrm{C}$ promoter drives myeloid-specific expression in transgenic fish. BMC Dev. Biol. 7:42. doi: 10.1186/1471-213X-7-42

Han, S. H., Einstein, G., Weisgraber, K. H., Strittmatter, W. J., Saunders, A. M., Pericak-Vance, M., et al. (1994). Apolipoprotein E is localized to the cytoplasm of human cortical neurons: a light and electron microscopic study. J. Neuropathol. Exp. Neurol. 53, 535-544. doi: 10.1097/00005072-19940900000013

Hasegawa, T., Hall, C. J., Crosier, P. S., Abe, G., Kawakami, K., Kudo, A., et al. (2017). Transient inflammatory response mediated by interleukin-1beta is required for proper regeneration in zebrafish fin fold. eLife 6:e22716. doi: 10 . 7554/eLife. 22716

Hashimoto, D., Chow, A., Noizat, C., Teo, P., Beasley, M. B., Leboeuf, M., et al. (2013). Tissue-resident macrophages self-maintain locally throughout adult life with minimal contribution from circulating monocytes. Immunity 38, 792-804. doi: 10.1016/j.immuni.2013.04.004

He, S., Chen, J., Jiang, Y., Wu, Y., Zhu, L., Jin, W., et al. (2018). Adult zebrafish Langerhans cells arise from hematopoietic stem/progenitor cells. eLife 7:e36131. doi: 10.7554/eLife.36131

Henriques, C. M., Carneiro, M. C., Tenente, I. M., Jacinto, A., and Ferreira, M. G. (2013). Telomerase is required for zebrafish lifespan. PLoS Genet. 9:e1003214. doi: 10.1371/journal.pgen.1003214

Herbomel, P., Thisse, B., and Thisse, C. (2001). Zebrafish early macrophages colonize cephalic mesenchyme and developing brain, retina, and epidermis through a M-CSF receptor-dependent invasive process. Dev. Biol. 238, 274-288. doi: 10.1006/dbio.2001.0393

Hernandez, P. P., Strzelecka, P. M., Athanasiadis, E. I., Hall, D., Robalo, A. F., Collins, C. M., et al. (2018). Single-cell transcriptional analysis reveals ILClike cells in zebrafish. Sci. Immunol. 3:eaau5265. doi: 10.1126/sciimmunol. aau 5265

Hitchcock, P. F., and Raymond, P. A. (2004). The teleost retina as a model for developmental and regeneration biology. Zebrafish 1, 257-271. doi: 10.1089/ zeb.2004.1.257

Hoeffel, G., and Ginhoux, F. (2015). Ontogeny of tissue-resident macrophages. Front. Immunol. 6:486. doi: 10.3389/fimmu.2015.00486

Hoeffel, G., Wang, Y., Greter, M., See, P., Teo, P., Malleret, B., et al. (2012). Adult Langerhans cells derive predominantly from embryonic fetal liver monocytes with a minor contribution of yolk sac-derived macrophages. J. Exp. Med. 209, 1167-1181. doi: 10.1084/jem.20120340

Holtmaat, A., and Caroni, P. (2016). Functional and structural underpinnings of neuronal assembly formation in learning. Nat. Neurosci. 19, 1553-1562. doi: 10.1038/nn.4418

Hsu, K., Traver, D., Kutok, J. L., Hagen, A., Liu, T. X., Paw, B. H., et al. (2004). The pu.1 promoter drives myeloid gene expression in zebrafish. Blood 104, 1291-1297. doi: 10.1182/blood-2003-09-3105

Hui, S. P., Sheng, D. Z., Sugimoto, K., Gonzalez-Rajal, A., Nakagawa, S., Hesselson, D., et al. (2017). Zebrafish regulatory T cells mediate organ-specific regenerative programs. Dev. Cell 43, 659-672.e5. doi: 10.1016/j.devcel.2017. 11.010

Hulsmans, M., Clauss, S., Xiao, L., Aguirre, A. D., King, K. R., Hanley, A., et al. (2017). Macrophages facilitate electrical conduction in the heart. Cell 169, 510-522.e20. doi: 10.1016/j.cell.2017.03.050

Jadhav, A. P., Roesch, K., and Cepko, C. L. (2009). Development and neurogenic potential of Muller glial cells in the vertebrate retina. Prog. Retin. Eye Res. 28, 249-262. doi: 10.1016/j.preteyeres.2009.05.002

Jelcic, I., Nimer, F. A. L., Wang, J., Lentsch, V., Planas, R., Jelcic, I., et al. (2018). Memory B cells activate brain-homing, autoreactive $\mathrm{CD}^{+} \mathrm{T}$ cells in multiple sclerosis. Cell 175, 85-100.e23. doi: 10.1016/j.cell.2018.08.011

Kanwal, Z., Wiegertjes, G. F., Veneman, W. J., Meijer, A. H., and Spaink, H. P. (2014). Comparative studies of Toll-like receptor signalling using zebrafish. Dev. Comp. Immunol. 46, 35-52. doi: 10.1016/j.dci.2014.02.003

Kroehne, V., Freudenreich, D., Hans, S., Kaslin, J., and Brand, M. (2011). Regeneration of the adult zebrafish brain from neurogenic radial glia-type progenitors. Development 138, 4831-4841. doi: 10.1242/dev.072587

Kumar, A., Pandey, R. K., Miller, L. J., Singh, P. K., and Kanwar, M. (2013). Muller glia in retinal innate immunity: a perspective on their roles in endophthalmitis. Crit. Rev. Immunol. 33, 119-135. doi: 10.1615/CritRevImmunol.201300 6618 
Kyritsis, N., Kizil, C., Zocher, S., Kroehne, V., Kaslin, J., Freudenreich, D., et al. (2012). Acute inflammation initiates the regenerative response in the adult zebrafish brain. Science 338, 1353-1356. doi: 10.1126/science.1228773

Lai, S. L., Marin-Juez, R., Moura, P. L., Kuenne, C., Lai, J. K. H., Tsedeke, A. T., et al. (2017). Reciprocal analyses in zebrafish and medaka reveal that harnessing the immune response promotes cardiac regeneration. eLife 6:e25605. doi: 10.7554/ eLife. 25605

Lam, S. H., Chua, H. L., Gong, Z., Lam, T. J., and Sin, Y. M. (2004). Development and maturation of the immune system in zebrafish, Danio rerio: a gene expression profiling, in situ hybridization and immunological study. Dev. Comp. Immunol. 28, 9-28. doi: 10.1016/S0145-305X(03)00103-4

Langenau, D. M., Ferrando, A. A., Traver, D., Kutok, J. L., Hezel, J. P., Kanki, J. P., et al. (2004). In vivo tracking of T cell development, ablation, and engraftment in transgenic zebrafish. Proc. Natl. Acad. Sci. U.S.A. 101, 7369-7374. doi: 10. 1073/pnas.0402248101

Langenau, D. M., Traver, D., Ferrando, A. A., Kutok, J., Aster, J. C., Kanki, J. P., et al. (2003). Myc-induced T-Cell leukemia in transgenic zebrafish. Science 299, 887-890. doi: 10.1126/science. 1080280

Lavine, K. J., Epelman, S., Uchida, K., Weber, K. J., Nichols, C. G., Schilling, J. D., et al. (2014). Distinct macrophage lineages contribute to disparate patterns of cardiac recovery and remodeling in the neonatal and adult heart. Proc. Natl. Acad. Sci. U.S.A. 111, 16029-16034. doi: 10.1073/pnas.1406508111

Lewis, K. L., Del Cid, N., and Traver, D. (2014). Perspectives on antigen presenting cells in zebrafish. Dev. Comp. Immunol. 46, 63-73. doi: 10.1016/j.dci.2014. 03.010

Liu, X., Li, Y. S., Shinton, S. A., Rhodes, J., Tang, L., Feng, H., et al. (2017). Zebrafish B cell development without a pre-b cell stage, revealed by CD79 fluorescence reporter transgenes. J. Immunol. 199, 1706-1715. doi: 10.4049/ jimmunol.1700552

Liu, Y. H., Ding, Y., Gao, C. C., Li, L. S., Wang, X. Y., and Xu, J. D. (2018). Functional macrophages and gastrointestinal disorders. World J. Gastroenterol. 24, 1181-1195. doi: 10.3748/wjg.v24.i11.1181

London, A., Benhar, I., and Schwartz, M. (2013). The retina as a window to the brain-from eye research to CNS disorders. Nat. Rev. Neurol. 9, 44-53. doi: 10.1038/nrneurol.2012.227

Lu, J., Peatman, E., Tang, H., Lewis, J., and Liu, Z. (2012). Profiling of gene duplication patterns of sequenced teleost genomes: evidence for rapid lineagespecific genome expansion mediated by recent tandem duplications. BMC Genomics 13:246. doi: 10.1186/1471-2164-13-246

Lucin, K. M., and Wyss-Coray, T. (2009). Immune activation in brain aging and neurodegeneration: too much or too little? Neuron 64, 110-122. doi: 10.1016/j. neuron.2009.08.039

Lugo-Villarino, G., Balla, K. M., Stachura, D. L., Banuelos, K., Werneck, M. B., and Traver, D. (2010). Identification of dendritic antigen-presenting cells in the zebrafish. Proc. Natl. Acad. Sci. U.S.A. 107, 15850-15855. doi: 10.1073/pnas. 1000494107

Lyons, D. A., and Talbot, W. S. (2014). Glial cell development and function in zebrafish. Cold Spring Harb. Perspect. Biol. 7:a020586. doi: 10.1101/cshperspect. a020586

Malvin, N. P., Seno, H., and Stappenbeck, T. S. (2012). Colonic epithelial response to injury requires Myd88 signaling in myeloid cells. Mucosal Immunol. 5, 194-206. doi: 10.1038/mi.2011.65

Man, A. L., Gicheva, N., and Nicoletti, C. (2014). The impact of ageing on the intestinal epithelial barrier and immune system. Cell. Immunol. 289, 112-118. doi: 10.1016/j.cellimm.2014.04.001

Marjoram, L., Alvers, A., Deerhake, M. E., Bagwell, J., Mankiewicz, J., Cocchiaro, J. L., et al. (2015). Epigenetic control of intestinal barrier function and inflammation in zebrafish. Proc. Natl. Acad. Sci. U.S.A. 112, 2770-2775. doi: 10.1073/pnas.1424089112

Marjoram, L., and Bagnat, M. (2015). Infection, inflammation and healing in zebrafish: intestinal inflammation. Curr. Pathobiol. Rep. 3, 147-153. doi: 10. 1007/s40139-015-0079-x

Martins, R. R., McCracken, A. W., Simons, M. J. P., Henriques, C. M., and Rera, M. (2018). How to catch a Smurf? in vivo assessment of intestinal permeability in multiple model organisms. Bio Protoc. 8:e2722. doi: 10.21769/BioProtoc.2722

Mathias, J. R., Dodd, M. E., Walters, K. B., Yoo, S. K., Erik, A., and Huttenlocher, A. (2010). Characterization of zebrafish larval inflammatory macrophages Jonathan. Dev. Comp. Immunol. 33, 1212-1217. doi: 10.1016/j. dci.2009.07.003

Mathias, J. R., Perrin, B. J., Liu, T. X., Kanki, J., Look, A. T., and Huttenlocher, A. J. (2006). Resolution of inflammation by retrograde chemotaxis of neutrophils in transgenic zebrafish. J. Leukoc. Biol. 80, 1281-1288. doi: 10.1189/jlb.0506346

Meijer, A. H., and Spaink, H. (2011). Host-pathogen interactions made transparent with the zebrafish model. Curr. Drug Targets 12, 1000-1017. doi: 10.2174/ 138945011795677809

Meijer, A. H., van der Vaart, M., and Spaink, H. P. (2014). Real-time imaging and genetic dissection of host-microbe interactions in zebrafish. Cell. Microbiol. 16, 39-49. doi: 10.1111/cmi.12236

Mitchell, D. M., Lovel, A. G., and Stenkamp, D. L. (2018). Dynamic changes in microglial and macrophage characteristics during degeneration and regeneration of the zebrafish retina. J. Neuroinflammation 15:163. doi: 10.1186/ s12974-018-1185-6

Moss, L. D., Monette, M. M., Jaso-Friedmann, L., Leary, J. H. III, Dougan, S. T., Krunkosky, T., et al. (2009). Identification of phagocytic cells, NK-like cytotoxic cell activity and the production of cellular exudates in the coelomic cavity of adult zebrafish. Dev. Comp. Immunol. 33, 1077-1087. doi: 10.1016/j.dci.2009. 05.009

Mowat, A. M. (2018). To respond or not to respond - a personal perspective of intestinal tolerance. Nat. Rev. Immunol. 18, 405-415. doi: 10.1038/s41577-0180002-x

Mowat, A. M., Scott, C. L., and Bain, C. C. (2017). Barrier-tissue macrophages: functional adaptation to environmental challenges. Nat. Med. 23, 1258-1270. doi: $10.1038 / \mathrm{nm} .4430$

Ng, A. N., de Jong-Curtain, T. A., Mawdsley, D. J., White, S. J., Shin, J., Appel, B., et al. (2005). Formation of the digestive system in zebrafish: III. Intestinal epithelium morphogenesis. Dev. Biol. 286, 114-135. doi: 10.1016/j.ydbio.2005. 07.013

Nguyen-Chi, M., Laplace-Builhe, B., Travnickova, J., Luz-Crawford, P., Tejedor, G., Phan, Q. T., et al. (2015). Identification of polarized macrophage subsets in zebrafish. eLife 4:e07288. doi: 10.7554/eLife.07288

Ogryzko, N. V., Lewis, A., Wilson, H. L., Meijer, A. H., Renshaw, S. A., and Elks, P. M. (2018). Hif- $1 \alpha$-induced expression of Il- $1 \beta$ protects against mycobacterial infection in zebrafish. J. Immunol. 202, 494-502. doi: 10.4049/jimmunol. 1801139

Oosterhof, N., Boddeke, E., and van Ham, T. J. (2015). Immune cell dynamics in the CNS: learning from the zebrafish. Glia 63, 719-735. doi: 10.1002/glia.22780

Oosterhof, N., Holtman, I. R., Kuil, L. E., van der Linde, H. C., Boddeke, E. W., Eggen, B. J., et al. (2017). Identification of a conserved and acute neurodegeneration-specific microglial transcriptome in the zebrafish. Glia 65, 138-149. doi: 10.1002/glia.23083

Page, D. M., Wittamer, V., Bertrand, J. Y., Lewis, K. L., Pratt, D. N., Delgado, N., et al. (2013). An evolutionarily conserved program of B-cell development and activation in zebrafish. Blood 122, e1-e11. doi: 10.1182/blood-2012-12471029

Perdiguero, E. G., Klapproth, K., Schulz, C., Busch, K., de Bruijn, M., Rodewald, H. R., et al. (2015). The origin of tissue-resident macrophages: when an erythromyeloid progenitor is an erythro-myeloid progenitor. Immunity 43, 1023-1024. doi: 10.1016/j.immuni.2015.11.022

Pereiro, P., Varela, M., Diaz-Rosales, P., Romero, A., Dios, S., Figueras, A., et al. (2015). Zebrafish Nk-lysins: first insights about their cellular and functional diversification. Dev. Comp. Immunol. 51, 148-159. doi: 10.1016/j.dci.2015. 03.009

Peri, F., and Nusslein-Volhard, C. (2008). Live imaging of neuronal degradation by microglia reveals a role for v0-ATPase al in phagosomal fusion in vivo. Cell 133, 916-927. doi: 10.1016/j.cell.2008.04.037

Poirier, J., Hess, M., May, P. C., and Finch, C. E. (1991). Astrocytic apolipoprotein E mRNA and GFAP mRNA in hippocampus after entorhinal cortex lesioning. Brain Res. Mol. Brain Res. 11, 97-106. doi: 10.1016/0169-328X(91)90111-A

Pope, H., and Voigt, M. M. (2014). Peripheral glia have a pivotal role in the initial response to axon degeneration of peripheral sensory neurons in zebrafish. PLoS One 9:e103283. doi: 10.1371/journal.pone.0103283

Pott, J., and Hornef, M. (2012). Innate immune signalling at the intestinal epithelium in homeostasis and disease. EMBO Rep. 13, 684-698. doi: 10.1038/ embor.2012.96 
Ramirez, A. I., de Hoz, R., Salobrar-Garcia, E., Salazar, J. J., Rojas, B., Ajoy, D., et al. (2017). The role of microglia in retinal neurodegeneration: Alzheimer's disease, Parkinson, and glaucoma. Front. Aging Neurosci. 9:214. doi: 10.3389/fnagi.2017. 00214

Redd, M. J., Kelly, G., Dunn, G., Way, M., and Martin, P. (2006). Imaging macrophage chemotaxis in vivo: studies of microtubule function in zebrafish wound inflammation. Cell Motil. Cytoskeleton 63, 415-422. doi: 10.1002/cm. 20133

Reichenbach, A., and Bringmann, A. (2013). New functions of Muller cells. Glia 61, 651-678. doi: 10.1002/glia.22477

Renshaw, S. A., Loynes, C. A., Trushell, D. M., Elworthy, S., Ingham, P. W., and Whyte, M. K. (2006). A transgenic zebrafish model of neutrophilic inflammation. Blood 108, 3976-3978. doi: 10.1182/blood-2006-05024075

Renshaw, S. A., and Trede, N. S. (2012). A model 450 million years in the making: zebrafish and vertebrate immunity. Dis. Model. Mech. 5, 38-47. doi: 10.1242/ dmm. 007138

Sato, S., Kiyono, H., and Fujihashi, K. (2015). Mucosal immunosenescence in the gastrointestinal tract: a mini-review. Gerontology 61, 336-342. doi: 10.1159/ 000368897

Schnabel, K., Wu, C. C., Kurth, T., and Weidinger, G. (2011). Regeneration of cryoinjury induced necrotic heart lesions in zebrafish is associated with epicardial activation and cardiomyocyte proliferation. PLoS One 6:e18503. doi: 10.1371/journal.pone.0018503

Sepulcre, M. P., Alcaraz-Perez, F., Lopez-Munoz, A., Roca, F. J., Meseguer, J., Cayuela, M. L., et al. (2009). Evolution of lipopolysaccharide (LPS) recognition and signaling: fish TLR4 does not recognize LPS and negatively regulates NF-kappaB activation. J. Immunol. 182, 1836-1845. doi: 10.4049/jimmunol. 0801755

Sheng, J., Ruedl, C., and Karjalainen, K. (2015). Most tissue-resident macrophages except microglia are derived from fetal hematopoietic stem cells. Immunity 43, 382-393. doi: 10.1016/j.immuni.2015.07.016

Shiau, C. E., Monk, K. R., Joo, W., and Talbot, W. S. (2013). An anti-inflammatory NOD-like receptor is required for microglia development. Cell Rep. 5, 13421352. doi: 10.1016/j.celrep.2013.11.004

Silverman, S. M., and Wong, W. T. (2018). Microglia in the retina: roles in development, maturity, and disease. Annu. Rev. Vis. Sci. 4, 45-77. doi: 10.1146/ annurev-vision-091517-034425

Skaggs, K., Goldman, D., and Parent, J. M. (2014). Excitotoxic brain injury in adult zebrafish stimulates neurogenesis and long-distance neuronal integration. Glia 62, 2061-2079. doi: 10.1002/glia.22726

Soenen, S., Rayner, C. K., Jones, K. L., and Horowitz, M. (2016). The ageing gastrointestinal tract. Curr. Opin. Clin. Nutr. Metab. Care 19, 12-18. doi: 10. 1097/MCO.0000000000000238

Stoll, G., Meuller, H. W., Trapp, B. D., and Griffin, J. W. (1989). Oligodendrocytes but not astrocytes express apolipoprotein E after injury of rat optic nerve. Glia 2, 170-176. doi: 10.1002/glia.440020306

Svahn, A. J., Graeber, M. B., Ellett, F., Lieschke, G. J., Rinkwitz, S., Bennett, M. R., et al. (2013). Development of ramified microglia from early macrophages in the zebrafish optic tectum. Dev. Neurobiol. 73, 60-71. doi: 10.1002/dneu.22039

Tang, Q., Iyer, S., Lobbardi, R., Moore, J. C., Chen, H., Lareau, C., et al. (2017). Dissecting hematopoietic and renal cell heterogeneity in adult zebrafish at single-cell resolution using RNA sequencing. J. Exp. Med. 214, 2875-2887. doi: $10.1084 /$ jem. 20170976

Traver, D., Paw, B. H., Poss, K. D., Penberthy, W. T., Lin, S., and Zon, L. I. (2003). Transplantation and in vivo imaging of multilineage engraftment in zebrafish bloodless mutants. Nat. Immunol. 4, 1238-1246. doi: 10.1038/ni1007

Trede, N. S., Langenau, D. M., Traver, D., Look, A. T., and Zon, L. I. (2004). The use of zebrafish to understand immunity. Immunity 20, 367-379. doi: 10.1016/S1074-7613(04)00084-6

van Ham, T. J., Brady, C. A., Kalicharan, R. D., Oosterhof, N., Kuipers, J., VeenstraAlgra, A., et al. (2014). Intravital correlated microscopy reveals differential macrophage and microglial dynamics during resolution of neuroinflammation. Dis. Model. Mech. 7, 857-869. doi: 10.1242/dmm.014886

Van Houcke, J., Bollaerts, I., Geeraerts, E., Davis, B., Beckers, A., Van Hove, I., et al. (2017). Successful optic nerve regeneration in the senescent zebrafish despite age-related decline of cell intrinsic and extrinsic response processes. Neurobiol. Aging 60, 1-10. doi: 10.1016/j.neurobiolaging.2017.08.013

Vecino, E., Rodriguez, F. D., Ruzafa, N., Pereiro, X., and Sharma, S. C. (2016). Glianeuron interactions in the mammalian retina. Prog. Retin. Eye Res. 51, 1-40. doi: 10.1016/j.preteyeres.2015.06.003

Wallace, K. N., Akhter, S., Smith, E. M., Lorent, K., and Pack, M. (2005). Intestinal growth and differentiation in zebrafish. Mech. Dev. 122, 157-173. doi: 10.1016/ j.mod.2004.10.009

Walton, E. M., Cronan, M. R., Beerman, R. W., and Tobin, D. M. (2015). The macrophage-specific promoter mfap4 allows live, long-term analysis of macrophage behavior during mycobacterial infection in zebrafish. PLoS One 10:e0138949. doi: 10.1371/journal.pone.0138949

Wang, M., Ma, W., Zhao, L., Fariss, R. N., and Wong, W. T. (2011). Adaptive Muller cell responses to microglial activation mediate neuroprotection and coordinate inflammation in the retina. J. Neuroinflammation 8:173. doi: 10. 1186/1742-2094-8-173

Wang, M., Wang, X., Zhao, L., Ma, W., Rodriguez, I. R., Fariss, R., et al. (2014). Macroglia-microglia interactions via TSPO signaling regulates microglial activation in the mouse retina. J. Neurosci. 34, 3793-3806. doi: 10.1523/ JNEUROSCI.3153-13.2014

White, D. T., Sengupta, S., Saxena, M. T., Xu, Q., Hanes, J., Ding, D., et al. (2017). Immunomodulation-accelerated neuronal regeneration following selective rod photoreceptor cell ablation in the zebrafish retina. Proc. Natl. Acad. Sci. U.S.A. 114, E3719-E3728. doi: 10.1073/pnas.1617721114

Wittamer, V., Bertrand, J. Y., Gutschow, P. W., and Traver, D. (2011). Characterization of the mononuclear phagocyte system in zebrafish. Blood 117, 7126-7135. doi: 10.1182/blood-2010-11-321448

Xu, J., Zhu, L., He, S., Wu, Y., Jin, W., Yu, T., et al. (2015). Temporalspatial resolution fate mapping reveals distinct origins for embryonic and adult microglia in zebrafish. Dev. Cell 34, 632-641. doi: 10.1016/j.devcel.2015. 08.018

Xu, Q., Bernardo, A., Walker, D., Kanegawa, T., Mahley, R. W., and Huang, Y. (2006). Profile and regulation of apolipoprotein E (ApoE) expression in the CNS in mice with targeting of green fluorescent protein gene to the ApoE locus. J. Neurosci. 26, 4985-4994. doi: 10.1523/JNEUROSCI.5476-05. 2006

Yoder, J. A., Turner, P. M., Wright, P. D., Wittamer, V., Bertrand, J. Y., Traver, D., et al. (2010). Developmental and tissue-specific expression of NITRs. Immunogenetics 62, 117-122. doi: 10.1007/s00251-009-0416-5

Yona, S., Kim, K.-W., Wolf, Y., Mildner, A., Varol, D., Breker, M., et al. (2013). Fate mapping reveals origins and dynamics of monocytes and tissue macrophages under homeostasis. Immunity 38, 79-91. doi: 10.1016/j.immuni.2012. 12.001

Zou, S., Tian, C., Ge, S., and Hu, B. (2013). Neurogenesis of retinal ganglion cells is not essential to visual functional recovery after optic nerve injury in adult zebrafish. PLoS One 8:e57280. doi: 10.1371/journal.pone.0057280

Conflict of Interest Statement: The authors declare that the research was conducted in the absence of any commercial or financial relationships that could be construed as a potential conflict of interest.

Copyright (c) 2019 Martins, Ellis, MacDonald, Richardson and Henriques. This is an open-access article distributed under the terms of the Creative Commons Attribution License (CC BY). The use, distribution or reproduction in other forums is permitted, provided the original author(s) and the copyright owner(s) are credited and that the original publication in this journal is cited, in accordance with accepted academic practice. No use, distribution or reproduction is permitted which does not comply with these terms. 